\title{
Modelling the term structure of interest rates á la Heath-Jarrow-Morton but with non Gaussian fluctuations
}

\author{
Przemysław Repetowicz ${ }^{\mathrm{a}}$ Brian Lucey ${ }^{\mathrm{b}}$ Peter Richmond ${ }^{\mathrm{a}}$ \\ ${ }^{a}$ Department of Physics, Trinity College Dublin 2, Ireland \\ ${ }^{\mathrm{b}}$ University of Dublin - School of Business Studies; University of Dublin - Institute \\ for International Integration Studies (IIIS)
}

\begin{abstract}
We consider a generalization of the Heath Jarrow Morton model for the term structure of interest rates where the forward rate is driven by Paretian fluctuations. We derive a generalization of Itô's lemma for the calculation of a differential of a Paretian stochastic variable and use it to derive a Stochastic Differential Equation for the discounted bond price. We show that it is not possible to choose the parameters of the model to ensure absence of drift of the discounted bond price. Then we consider a Continuous Time Random Walk with jumps driven by Paretian random variables and we derive the large time scaling limit of the jump probability distribution function (pdf). We show that under certain conditions defined in text the large time scaling limit of the jump pdf in the Fourier domain is $\tilde{\omega}_{t}(k, t) \sim \exp \left\{-\mathfrak{K} /(\ln (k t))^{2}\right\}$ and is different from the case of a random walk with Gaussian fluctuations.
\end{abstract}

Key words: Stochastic differential equations, Itô's lemma, Term structure of interest rates, Pricing of financial derivatives, Scaling limits of Continuous Time Random Walks, Steepest descent approximation PACS:02.50.-r Probability theory, stochastic processes, and statistics, 05.40.-a Fluctuation phenomena, random processes, noise, and Brownian motion, 89.90.+n Other topics of general interest to physicists

Email addresses: repetowp@tcd.ie (Przemysław Repetowicz), blucey@tcd.ie (Brian Lucey), richmond@tcd.ie (Peter Richmond).

URLs: www.maths.tcd.ie/ przemek (Przemysław Repetowicz), www.tcd.ie/Physics/People/Peter.Richmond (Peter Richmond).

Preprint submitted to Elsevier Science 30 October 2018 


\section{Introduction}

The Arbitrage Pricing Theory (APT) is a generalization of the Capital Asset Pricing Model (CAPM), and it states that capital assets are priced according to a set of factors. It does not say what these factors are, just that only idiosyncratic (firm specific) risk is priced. So, two different stocks could have two different APT formulations.

However, the law of one price states that two identical assets should have the same value on the financial market and deviations from rational behavior of traders for example, significantly larger returns on Fridays the $13^{\text {th }}$, Lucev (2000) have been evidenced. Yet, market efficiency is an assumed prerequisite for CAPM to hold and contemporary finance still uses APT as a quantitative tool. Heath-Jarrow-Morton (1992) (see also Musiela-Rutkowski (1991)) have formulated a general theory (to be referred to as the HJM model) and a unifying framework for the existing APTs.

Presuming the forward interest rate and the bond price to be a continuous time stochastic process described by a Stochastic Differential Equation (SDE) they derive the generic form of the SDE that ensures that the bond price, related to the zero coupon bond (risk-less security issued by the central bank at a fixed interest rate called the spot rate), has a mean zero as a function of time.

The HJM model has been generalized by Sornette (1998), who, drawing on some ideas from string theory, derives a general condition for a class of second order SDEs that must be satisfied to ensure absence of arbitrage opportunities. He obtains a whole class of, nonlinear second order SDEs that, in some cases, can be solved exactly and that also provide extensions to the theory of pricing options Musiela-Rutkowski (1991).

These models have a rich structure but are based on Gaussian fluctuations. In this paper we develop a corresponding theory that allows the fluctuations to have an arbitrary distribution, in particular they may have power-law behavior at the high end.

\section{Theoretical analysis}

The purpose of this section is to provide overall understanding of stochastic processes that involve asymptotically large, power-law distributed, fluctuations. The analysis lacks mathematical rigor and draws more on physical ideas of Langévin processes (even though they are not explicitly mentioned in 
the text) than on the abstract theory of stochastic processes. The derivation of strict mathematical foundations for the analysis (e.g. foundations for the Lemma and the Statement in the next section) is left for future work.

\subsection{Definitions and heuristic reasoning}

The forward interest rate $f(t, T)$ is an interest rate that is agreed at time $t$ and that gives the trader a right to trade in an asset at time $T$ (to be termed as maturity). The underlying assumption in the HJM model is to describe $f(t ; T)$ through a following SDE:

$$
f(t+\epsilon ; T)=f(t ; T)+\int_{t}^{t+\epsilon} \alpha(\theta ; T) d \theta+\int_{t}^{t+\epsilon} \sigma(\theta ; T) \dot{\eta}(\theta) d \theta
$$

where $\epsilon>0$. Here the functions $\alpha$ and $\sigma$, called drift and volatility are in general dependent on the process itself, and the fluctuations $\delta \eta=\dot{\eta}(\theta) d \theta$ have a zero mean, are uncorrelated with one another for different times and conform to a generic, non-Gaussian distribution with a density $\rho(\delta \eta)$. An asset which at maturity has a value one Euro has at some earlier time $t$ a smaller value that is expressed through a discount factor involving the forward rate (1) as follows:

$$
B(t ; T)=B(t, f(t, T) ; T)=\exp \left\{-\int_{t}^{T} f(t ; \xi)\right\} d \xi
$$

The bond price is related to the price of a zero-coupon bond $M(t)$ whose stochastic dynamics is given by a SDE that involves the spot rate of interest $f(\theta, \theta)$ and some fluctuations:

$$
M(t)=\exp \left\{-\int_{0}^{t} f(\theta, \theta) d \theta+\int_{0}^{t} \dot{\eta}(\theta) d \theta\right\}
$$

and conditions are derived for the discounted bond price $M(t) B(t ; T)$ to have a zero drift in order to ensure absence of arbitrage opportunities. The zerodrift condition is also referred to as the "martingale property" and states that the conditional mean of the bond price at time $s>t$ discounted back to time zero equals the discounted bond price at time $t$. Here $E\left[M(s) B(s ; T) \mid \mathcal{F}_{t}\right]=$ $M(t) B(t ; T)$ and the conditioning is on the so called "filtration" $\mathcal{F}_{t}$ of information meaning a set of information about the bond price up to time $t$ (see Musiela-Rutkowski (1991)). 
The main problem is now to derive a SDE for the bond price (2). For computing a differential of a function of Gaussian fluctuations one can use the lemma by Ito (1997). This lemma states that the differential is obtained by expanding the function into a Taylor series to the first order in time and to the second order in the fluctuations and by replacing differentials of the fluctuations via their averages. If the function of the stochastic process $f$ has a form given by equation (2) we may use a theorem by Girsanov [see Musiela-Rutkowski (1991)] that simplifies the computation.

If, however, the fluctuations are not Gaussian, in particular if the likelihood for them to be large is much larger than in the Gaussian case then the higher moments and auto-covariances of the process may be infinite (as in the case of a Lévy or a Pareto distribution) and the procedure for computing the differential may fail. It may also happen that the probability distribution of the squared fluctuation is of the same kind as that of the fluctuation itself which will certainly change the structure of the SDE that describes the bond price (2). In the following we examine the mathematical difficulties that arise. Since we are not aware of any generalization of Itô's lemma that can be used in a straightforward manner for the calculation of the differential of the bond price, we must perform the computations from scratch. The procedure consists in first working out a small change of the bond price, secondly in expressing it via the forward rate and finally in performing the limit $\epsilon \rightarrow 0$. Here the change reads :

$$
B(t+\epsilon ; T)-B(t ; T)=B(t ; T)\left[e^{\int_{t}^{t+\epsilon} f(t+\epsilon, \xi) d \xi} \exp \left(-\int_{t}^{T} \delta_{\epsilon} f(t ; \xi) d \xi\right)-1\right]
$$

where $\delta_{\epsilon} f(t ; \xi):=f(t+\epsilon ; \xi)-f(t ; \xi)$. Now we expand the right hand side in a Taylor series and retain terms proportional to $\epsilon$ neglecting higher powers of $\epsilon$. The expression in square brackets on the right hand side of (4) reads:

$$
\begin{aligned}
& \int_{t}^{t+\epsilon} f(t+\epsilon, \xi) d \xi+\sum_{n=1}^{\infty} \frac{(-1)^{n}}{n !}\left(-\int_{t}^{T} \delta_{\epsilon} f(t ; \xi) d \xi\right)^{n}= \\
& \int_{t}^{t+\epsilon} f(t+\epsilon, \xi) d \xi-\int_{t}^{T} \int_{t}^{t+\epsilon} \alpha(\theta ; \xi) d \theta d \xi-\int_{t}^{T} \int_{t}^{t+\epsilon} \sigma(\theta ; \xi) \dot{\eta}(\theta) d \theta d \xi \\
& +\sum_{n=2}^{\infty} \frac{(-1)^{n}}{n !}\left(\int_{t}^{T} \int_{t}^{t+\epsilon} \sigma(\theta, \xi) \dot{\eta}(\theta) d \theta d \xi\right)^{n}
\end{aligned}
$$

Now the whole problem consists in a proper treatment of the products of fluctuations $\prod_{i=1}^{n} \delta \eta\left(\theta_{i}\right)$ in the last term on the right hand side in (5). This is the point where the analysis differs essentially from the Gaussian case. We 
assume the fluctuations to conform to a Lévy (or Paretian) distribution. We set $\delta \eta=\operatorname{Lévy}_{\mu}(0,1,0) d t$ which means that $\dot{\eta}=\delta \eta / d t$ is (parameters in brackets from the left to the right) a Paretian distribution with a characteristic exponent $\mu$, skewness zero, dispersion one and mean zero (to be termed standard Lévy variable). The Paretian distribution has a pdf whose asymptotic expansion reads:

$$
\rho(\delta \eta)=\frac{1}{\pi} \sum_{n=1}^{\infty} \sin \left(\frac{\pi}{2} \mu n\right) \Gamma(\mu n+1) \frac{d t^{n}}{x^{\mu n+1}}
$$

From this expansion follows an important property for the product of many Paretian variables.

\section{Lemma:}

(A) If $\delta \eta=\operatorname{Lévy}_{\mu}(0,1,0) d t$ then $(\delta \eta)^{n}=\operatorname{Lévy}_{\mu / n}(0,1,0)(d t / n)$

(B) For independent Lévy variables $\delta \eta_{1}$ and $\delta \eta_{2}$ we have

$$
Z=\delta \eta_{1} \delta \eta_{2} \sim \operatorname{Lévy}_{\mu}(0,1,0)(d t)^{2} .
$$

Both statements regard the high end of the distribution.

\section{Proof:}

(A) From the conservation of elementary probabilities $f_{Y}(y) d y=f_{X}(x) d x$ it follows that the pdf of the $n$-th power $Y=X^{n}$ of a Paretian variable $X=\operatorname{Lévy}_{\mu}(0,1,0) d t$ reads:

$$
\begin{aligned}
& f_{Y}(y)=\frac{f_{X}(x)}{n x^{n-1}}=\frac{f_{X}\left(y^{1 / n}\right)}{n y^{1-1 / n}}=\frac{1}{n y^{1-1 / n}} \sum_{m=1}^{\infty} \frac{a_{m} d t^{m}}{\left(y^{1 / n}\right)^{\mu m+1}}=\frac{1}{n} \sum_{m=1}^{\infty} \frac{a_{m} d t^{m}}{y^{(\mu / n) m+1}} \\
& \Rightarrow Y \sim \frac{d t}{n} \operatorname{Lévy~}_{\mu / n}(0,1,0)
\end{aligned}
$$

where $a_{m}$ denote coefficients in the expansion (6) for the pdf of a Paretian variable.

(B) If $X_{1}, X_{2}=\operatorname{Lévy}_{\mu}(0,1,0) d t$ are independent Paretian variables then the pdf of their product $Z=X_{1} X_{2}$ reads:

$$
\begin{aligned}
& f_{Z}(z)=\int_{1}^{z} \int_{1}^{z} \delta\left(z-x_{1} x_{2}\right) f_{X}\left(x_{1}\right) f_{X}\left(x_{2}\right) d x_{1} d x_{2} \\
& =\int_{1}^{z} \frac{d x_{1}}{x_{1}} f_{X}\left(x_{1}\right) f_{X}\left(\frac{z}{x_{1}}\right)=\int_{1}^{z} \frac{d x_{1}}{x_{1}} \frac{\left(a_{1} d t\right)}{\xi^{\mu+1}} \frac{\left(a_{1} d t\right)}{(z / \xi)^{\mu+1}}=\frac{\left(a_{1} d t\right)^{2}}{z^{\mu+1}} \ln (z) \\
& \Rightarrow Z \sim(d t)^{2} \operatorname{Lévy}_{\mu}(0,1,0)
\end{aligned}
$$

where (8) holds only for large values of $z$ since we have replaced the

pdfs by the first term of the asymptotic expansion (6). From the last 
expression in (8) it follows that ,up to a logarithmic correction, $Z$ is a Paretian with a squared dispersion q.e.d.

The Lemma implies that the only factors proportional to the first power of $\epsilon$ in the last sum on the right hand side of equation (5) are those which contain "diagonal" terms of the kind $\left(\delta \eta\left(\theta_{i}\right)\right)^{n}$. Indeed from (B) it follows that the nondiagonal terms, ie those including products $\left(\delta \eta\left(\theta_{1}\right)\right)^{n_{1}}\left(\delta \eta\left(\theta_{2}\right)\right)^{n_{2}} \ldots\left(\delta \eta\left(\theta_{p}\right)\right)^{n_{p}}$ of differentials of $\eta$ evaluated at different values of $\theta$ will all be proportional to higher powers of $\epsilon$ (since they are products of independent Paretian random variables) and can, therefore, be neglected. We emphasize the difference to the Gaussian case:

Statement The $n$-th power of a Gaussian with variance $d t$ is a random variable with variance $d t^{n}$. The $n$th power of a Paretian with a dispersion $d t$ also has a dispersion $d t$.

We now obtain, after taking the limit $\epsilon \rightarrow 0$ the following SDE for the bond price:

$$
\begin{aligned}
\frac{d_{t} B(t, T)}{B(t, T)} & =f(t, t)-\int_{t}^{T} \alpha(t ; \xi) d \xi+\sum_{n=1}^{\infty} \frac{(-1)^{n}}{n !}\left(\int_{t}^{T} \sigma(t, \xi) d \xi\right)^{n}\left(\dot{\eta}_{\mu}(t)\right)^{n} \\
& =f(t, t)-\int_{t}^{T} \alpha(t ; \xi) d \xi+\exp \left[-\left(\int_{t}^{T} \sigma(t, \xi) d \xi\right) \dot{\eta}_{\mu}(t)\right]-1
\end{aligned}
$$

In the next step we carry out the same procedure for the discount factor $M(t)$ defined in (3). Expanding $M(t+\epsilon)-M(t)$ in a Taylor series to arbitrary order in the variable $\int_{0}^{t}(f(\theta, \theta)-\dot{\eta}(\theta) d \theta) d \theta$, as in (5), we notice that after expanding the $n$-th (with $n>2$ ) power of the integral we get some terms that contain $f(\theta, \theta)$ and other terms that contain only the fluctuations $\dot{\eta}(\theta)$. The former terms will be of order $\epsilon^{2}$ and may be neglected. The only term left then is the $n$th power of the integral over fluctuations. This we rewrite as a sum of increments $\delta \eta(t+i d t)$ at times $(t+i d t)$. This corresponds to a division of nodes of the interval $[t, t+\epsilon]$ into $\epsilon / d t$ bins. Thus we have:

$$
\left(\int_{t}^{t+\epsilon} \dot{\eta}(\theta) d \theta\right)^{n}=\prod_{j=1}^{n}\left[\sum_{i_{j}=1}^{\epsilon / d t} \delta \eta\left(t+i_{j} d t\right)\right]=\sum_{i=1}^{\epsilon / d t}(\delta \eta(t+j d t))^{n}+O\left(\epsilon^{2}\right)=(\dot{\eta}(t))^{n} \epsilon+O\left(\epsilon^{2}\right)(11
$$

Now notice that on the grounds of Lemma and/or of the Statement that the power terms $(\delta \eta(t+j d t))^{n}$ are $d t / n \operatorname{Lévy}_{\mu / n}(0,1,0)$ whereas all other terms $\delta \eta\left(t+j_{1} d t\right)^{n_{1}} \cdot \ldots \cdot \delta \eta\left(t+j_{p} d t\right)^{n_{p}}$ that involve $p \geq 2$ different time values are, since they are products of $p$ independent Paretian variables, on the grounds 
of Lemma (B) of order of $d t^{p}$. Since their number is a polynomial of order $(\epsilon / d t)^{p}$ the total will be of order $\epsilon^{p}$ and can, therefore, be neglected.

In the limit $\epsilon \rightarrow 0$ only the powers of Paretian variables survive and we arrive at the following SDE for the discount factor:

$$
\frac{d_{t} M(t)}{M(t)}=-f(t, t)+\exp \left(\dot{\eta}_{\mu}(t)\right)-1
$$

Adding the logarithmic derivatives (12) and (10) we obtain the logarithmic derivative of the discounted bond price $V(t)=M(t) B(t ; T)$, ie the price at time zero of a bond that at maturity $T$ pays off one Euro and has, at some intermediate time $t$, the value $B(t ; T)$.

$$
\frac{d_{t} V(t)}{V(t)}=-\int_{t}^{T} \alpha(t ; \xi) d \xi-2+\exp \left[-\left(\int_{t}^{T} \sigma(t, \xi) d \xi\right) \dot{\eta}_{\mu}(t)\right]+\exp \left(\dot{\eta}_{\mu}(t)\right)(13
$$

This is an SDE for the discounted bond price that we must solve and then require the Martingale condition $E\left[V(s)-V(t) \mid \mathcal{F}_{t}\right]=0$ for every $s>t$ to be satisfied in order to ensure non-arbitrage opportunities in the market with non Gaussian fluctuations. Note that the time evolution of the logarithm of $V(t)$ is driven by two competing factors namely a negative "deterministic" drift corresponding to the first two terms on the right hand side in (13) and a positive fluctuations (the last two terms). Therefore the Martingale condition boils down to requiring the average fluctuation to be equal to the deterministic drift. Note also that, due to an exponential enhancement of the underlying fluctuations $\dot{\eta}_{\mu}(t)$, the fluctuations in (13) are very large; and huge when compared to Gaussian noise. It is, therefore, not clear whether the absence of arbitrage opportunities can be indeed ensured (see item (B) in the discussion section 2.2).

\subsection{Connections to other works in this subject matter}

At this stage we make some remarks regarding the connection (A),(B) of our result to works by other authors and to an abstract representation (C) of infinitely divisible distributions.

(A) The equations (10) and (13) cannot be directly related to the result (12) page 154 in Kleinert (2004). This is because the author assumes the $\operatorname{logarithmic}$ characteristic function $\phi(k):=\log E[\exp (i k \dot{\eta}(t))]$ of the fluctuations $\dot{\eta}(t)$ is an analytic function in $k$ or, in other words, that all moments of the fluctuations are finite $\left\langle(\dot{\eta}(t))^{p}\right\rangle<\infty$, (see expansion of 
the "Hamiltonian" - equation (6) page 153 in Kleinert (2004)) whereas we have $\phi(k)=\log E\left[i k \operatorname{Lévy}_{\mu}(0,1,0)\right]=-|k|^{\mu}$ which is not analytic as $k \rightarrow 0$ (some of the constants $c_{1}, c_{2}, \ldots$ in expansion (6) page 153 may be infinite).

(B) Bielecki (2004), using the framework of the abstract probability theory, considers a Gaussian HJM model with several forward interest rates, so called default-able $g_{i}(t ; T) i=1, \ldots, K$ rates and a default free rate $f(t ; T)$, subject to an ordering condition $g_{K}(t ; t)>\ldots>g_{1}(t ; t)>f(t ; t)$ on the respective spot rates of interest. They define a discounted Defaultable Corporate Bond (DCB) $\widehat{Z}(t ; T)$ that equals the consecutive discounted default-able bond prices $Z_{i}(t ; T)$. This is related to the defaultable forward rates of interest $g_{i}(t ; T)$, and the discounted default free rate of interest $f(t ; T)$ in time intervals $\tau_{i}<t<\tau_{i+1}$; intervals that are determined by the condition that the differences between the default-able bond prices $D_{i}(t ; T)$ and the default free $B(t ; T)$ price do not exceed a given threshold. In other words they consider a process of "credit migrations" where the DCB is taken as $D_{k}(t ; T)$ at time inception and is subsequently replaced by $D_{i}(t ; T)$ with $i<K$ once differences benchmarked on the default free price $B(t ; T)$ exceed a given threshold (see Fig.1 ). In addition, certain rates $\delta_{i} \in[0,1]$ of recovery (returns of losses from the Treasury Bond - central bank) are assumed in each time interval $t \in\left[\tau_{i}, \tau_{i+1}\right]$. Finally conditions on the absence of arbitrage opportunities for the DCB are deduced.

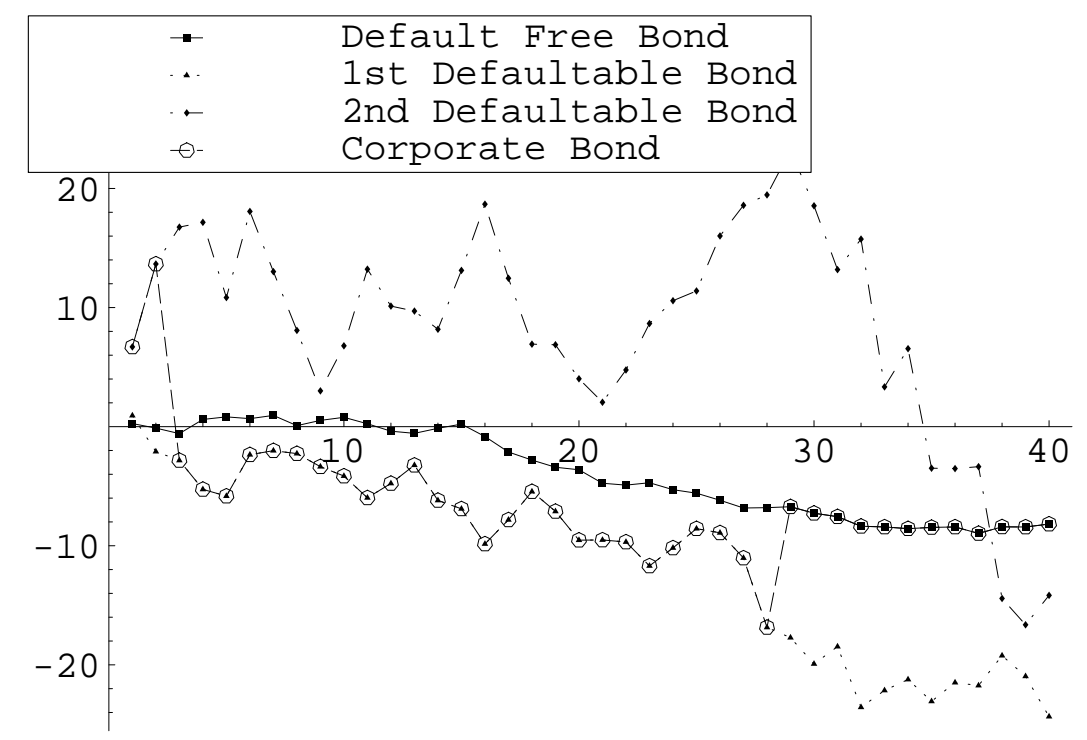

Fig. 1. Time evolution of the logarithm of price of the Discounted Corporate Bond as considered in Bielecki (2004). The evolution starts with the most highly default-able bond (Diamonds) and goes down to the less default-able (Triangles) and default free (Boxes) bonds once a threshold difference from the default free logarithm of price is reached. 
This is certainly a very sophisticated "real world" model yet it does not compare to our approach because in the presence of large Paretian fluctuations (see Statement), that are presumed in our approach, absence of arbitrage opportunities cannot be ensured. Indeed, a closer explanation of the time evolution (13) of the discounted bond price $V(t)$ ensures that it is impossible to choose the drift $\alpha(t ; T)$ and the volatility $\sigma(t ; T)$ such that the discounted bond price as a whole has a zero drift, ie the mean of a small increment $V(s)-V(t)$ for $s>t$ of the discounted bond price conditioned on the past history of the price process equals zero under a certain probability measure. This follows from a following "waving hand argument" . Due to an exponential enhancement of, already very strong, Paretian fluctuations; the mean of the right hand side of equation (13) is infinite. Therefore the positive "stochastic jumps" dictated by the last two terms in equation (13) are, for sufficiently large times $t$, arbitrarily large and cannot be offseted by any finite value of the negative "deterministic" drift coefficient $-\int_{t}^{T} \alpha(t ; \xi) d \xi-2$. Thus the discounted bond price drifts toward positive values like a biased random walk. Since it is impossible to damp the biased movement of the discounted bond price $V(t)$. Further theoretical analysis of the model will be pursued in a different direction namely we shall work out a distribution of random times needed for the biased movement to reach a certain threshold $\mathfrak{x}$ value of the log-price, known as first hitting times

$$
T_{\mathfrak{x}}=\min [t \mid \ln (V(t)=\mathfrak{x}]
$$

of the price. This can be done by writing down a Fokker-Planck differential equation, an equation that may include fractional derivatives Samko (1993); Meerschaert (2001) and that describes the spatio-temporal evolution of the probability for the discounted bond price to have a given value at a given time (to be termed occupation probability). The equation may be solved in the asymptotic limit of large times and the probability distribution of the first hitting times will be derived [Redner (2001)] from it. This approach will be pursued in section (3).

(C) Since the stochastic process (1) that describes the forward rate of interest is an Infinitely Divisible Distribution (IID) it must have a representation in terms of the Lévy-Khintchin formula (e.g. Theorem 3.1.11 page 41 in Meerschaert (2001)) for the log-characteristic function of an IID. Indeed the stochastic dynamics (1) of the forward rate can be considered as a a compound Poisson process $f(t ; T)=\alpha(t ; T)+\sigma(t ; T) \sum_{j=1}^{N_{t}} \delta \eta_{j}$ with $N_{t}=\operatorname{Poisson}\left(c_{\epsilon}\right)$ and $\delta \eta_{j}=\operatorname{Lévy}_{\mu}(0,1,0)$ with a very large rate $c_{\epsilon}$. This corresponds to taking a small positive $\epsilon>0$ and a measure $\phi(d x)$ in the Lévy-Khintchin formula equal to $\phi(d x) \sim 1 / x^{\alpha+1}$. In this case $c_{\epsilon}=\int_{\epsilon}^{\infty} d x / x^{\alpha+1}$ can be arbitrarily large for $\epsilon$ sufficiently small. Thus the jump pdf $\rho(\delta \eta) \sim c_{\epsilon}^{-1} 1 /(\delta \eta)^{\alpha+1}$ is related to a Paretian distribution for large values of the jump size $\delta \eta$. 


\section{Occupational probability of the discounted bond price}

Now we formulate an equation for the occupational probability $\mathcal{P}(x, t)=$ $P(\ln V(t)=x)$ for the logarithm $\ln V(t)$ of the discounted bond price in (13) to attain a value $x$ at time $t$. Note that since the process $\ln V(t)$ is a Continuous Time Random Walk (CTRW) with jumps occurring at random times the probability density function (pdf) $\phi_{n}(x, t)$ of reaching $x$ at time $t$ after having performed $n$ steps is a convolution of the pdf $\phi_{n-1}\left(x^{\prime}, t^{\prime}\right)$ and a jump probability $\omega_{t}\left(x-x^{\prime}, t-t^{\prime}\right)$. Here

$$
\phi_{n}(x, t)=\int_{\infty}^{\infty} \int_{0}^{t} \omega_{t}\left(x-x^{\prime}, t-t^{\prime}\right) \phi_{n-1}\left(x^{\prime}, t^{\prime}\right) d t^{\prime} d x^{\prime}
$$

Now we use the fact that the occupational probability $\mathcal{P}(x, t)$ is a convolution in time of the probability density of reaching $x$ at time $t^{\prime}$ after an arbitrary number of steps $n$ and the probability density of being unmoved in the time interval $\left[t^{\prime}, t\right]$. We obtain [Montroll (1965); Balescu (1997)] the following formula for the Fourier-Laplace transform $\tilde{P}(k, s):=\mathcal{F}_{x} \mathcal{L}_{t}[\mathcal{P}(x, t)](k, s)$ (with respect to $x$ and to time $t$ respectively) of the occupational probability.

$$
\tilde{P}(k, s)=\frac{1-\tilde{\phi}(s)}{s} \frac{1}{1-\tilde{\omega}_{t}(k, s)}
$$

where $\tilde{\omega}_{t}(k, s)=\mathcal{F}_{x} \mathcal{L}_{t}\left[\omega_{t}(x, t)\right]=\int_{-\infty}^{\infty} \int_{0}^{\infty} \exp (\imath k x-s t) \omega_{t}(x, t) d x d t$ is the Fourier-Laplace transform of the jump probability density and $\tilde{\phi}(s)=\tilde{\omega}_{t}(0, s)$ is the Laplace transform of the waiting time pdf. Thus in order to find the occupational probability we need to work out the Fourier-Laplace transform of the jump pdf.

Denoting $\Sigma:=\int_{t}^{T} \sigma(t, \xi) d \xi$ and $\Delta:=\int_{t}^{T} \alpha(t ; \xi) d \xi$ we express the jump probability as:

$$
\begin{aligned}
& \omega_{t}\left(x-x^{\prime}, t-t^{\prime}\right)=P\left(\ln V(t)-\ln V\left(t^{\prime}\right)=x-x^{\prime}\right) \\
& =P\left(\int_{t^{\prime}}^{t}\left(\exp \left[-\Sigma \dot{\eta}_{\mu}(\xi)\right]+\exp \left(\dot{\eta}_{\mu}(\xi)\right)\right) d \xi=x-x^{\prime}+\int_{t^{\prime}}^{t}(\Delta(\xi)+2) d \xi\right) \\
& =P\left(\frac{\Delta t}{N} \sum_{j=1}^{N}\left(\exp \left[-\Sigma \zeta_{j}\right]+\exp \left(\zeta_{j}\right)\right)=\left(x-x^{\prime}+\int_{t^{\prime}}^{t}(\Delta(\xi)+2) d \xi\right)\right) \\
& =\int_{-\infty}^{\infty} \ldots \int_{-\infty}^{\infty} \delta\left(\frac{\Delta t}{N} \sum_{j=1}^{N}\left(e^{-\Sigma \zeta_{j}}+e^{\zeta_{j}}\right)-\left(x-x^{\prime}+\int_{t^{\prime}}^{t}(\Delta(\xi)+2) d \xi\right)\right)\left[\prod_{j=1}^{N} \rho\left(\zeta_{j}\right) d \operatorname{dg}_{j} \rho\right]
\end{aligned}
$$


where the equality $(17)=(18)$ follows from the SDE (13). The next equality follows from the division of the time interval $\left[t^{\prime}, t\right]$ into $N$ intervals of length $\Delta t / N$ and from the definition of a Riemann integral. The final equality $(19)=(20)$, in which $\delta$ means the Dirac delta function, follows from the fact that the values of fluctuations $\zeta_{j}=\dot{\eta}_{\mu}\left(\xi_{j}\right)$ at intermediate times $\xi_{j}=t^{\prime}+j\left(t-t^{\prime}\right) / N$ are iid standard Lévy variables. Performing the Fourier transform of the jump pdf with respect to $x-x^{\prime}$ results in replacing the delta function in the integrand in (20) by an exponential $\exp \{\imath k \ldots\}$ and produces a following path integral

$$
\tilde{\omega}_{t}\left(k, t-t^{\prime}\right)=\frac{1}{2 \pi} \int_{-\infty}^{\infty} \ldots \int_{-\infty}^{\infty} \exp \left\{\imath k \int_{t^{\prime}}^{t} \mathcal{H}\left(\xi, \dot{\eta}_{\mu}(\xi)\right) d \xi\right\} D\left[\vec{\eta}_{\mu}\right]
$$

where the "Hamiltonian" $\mathcal{H}\left(\xi, \dot{\eta}_{\mu}(\xi)\right)$ reads:

$$
\begin{aligned}
\mathcal{H}\left(\xi, \dot{\eta}_{\mu}(\xi)\right) & =\mathcal{H}_{s}\left(\dot{\eta}_{\mu}(\xi)\right)-\mathcal{H}_{d}(\xi) \\
& =\left(\exp \left[-\Sigma \dot{\eta}_{\mu}(\xi)\right]+\exp \left(\dot{\eta}_{\mu}(\xi)\right)\right)-(\Delta(\xi)+2)
\end{aligned}
$$

Here we divided the "Hamiltonian" into two parts the "stochastic" part $\mathcal{H}_{s}\left(\dot{\eta}_{\mu}(\xi)\right)$ that depends on the fluctuations $\dot{\eta}_{\mu}(\xi)$ and the "deterministic" part $\mathcal{H}_{d}(\xi)$ that depends on the drift function $\Delta(\xi)$. The measure $D\left[\vec{\eta}_{\mu}\right]$ is expressed via pdfs of standard Lévy variables $\dot{\eta}_{\mu}\left(\xi_{j}\right)$ as follows: $D\left[\vec{\eta}_{\mu}\right]=$ $\lim _{N \rightarrow \infty}\left[\prod_{j=1}^{N} \rho\left(\dot{\eta}_{\mu}\left(\xi_{j}\right)\right) d \dot{\eta}_{\mu}\left(\xi_{j}\right)\right]$ Note that since the "Hamiltonian" depends only on the fluctuations $\dot{\eta}_{\mu}(\xi)$ and not on their time derivative $\ddot{\eta}_{\mu}(\xi)^{1}$ the path integral (21) is expressed via ordinary Riemann integrals only. Here:

$$
\tilde{\omega}_{t}\left(k, t-t^{\prime}\right)=\frac{1}{2 \pi} e^{-\imath k \int_{t^{\prime}}^{t} \mathcal{H}_{d}(\xi) d \xi} \cdot \lim _{N \rightarrow \infty}\left[\mathfrak{f}\left(\frac{k\left(t-t^{\prime}\right)}{\varpi(N)}\right)\right]^{N}
$$

where

$$
\mathfrak{f}(k):=\int_{-\infty}^{\infty} \exp \left\{\imath k \mathcal{H}_{s}(\zeta)\right\} \rho(\zeta) d \zeta=\int_{-\infty}^{\infty} \exp \left\{\imath k\left(e^{\zeta}+e^{-\Sigma \zeta}\right)\right\} \rho(\zeta) d \zeta
$$

and $\varpi(N)$ is a number of subintervals into which we split the interval $\left[t^{\prime}, t\right]$ so that the the path integral does not diverge when $N \rightarrow \infty$.

1 This would correspond to a propagator of the Schrödinger equation in quantum mechanics with a Hamiltonian that only depends on the coordinates of the particle and not on its velocities. 
In order to get additional insight into the solution of the problem in our further calculations we replace the integrand in the definition of $\mathfrak{f}(k)$ by a sum of two terms in which one of the $e^{(\ldots \zeta)}$ terms is neglected. The applicability of this approximation is yet to be examined.

\section{Approximation:}

$$
\mathfrak{f}(k) \simeq \frac{1}{2}(\mathfrak{F}(1, k)+\mathfrak{F}(-\Sigma, k))
$$

where

$$
\mathfrak{F}(a, k):=\int_{-\infty}^{\infty} \exp \left\{\imath k e^{a \zeta}\right\} \rho(\zeta) d \zeta=\frac{-1}{2 \pi a} \int_{-\infty}^{\infty} \exp \left(-|\xi|^{\mu}\right)\left(\frac{k}{\imath}\right)^{\imath \xi / a} \Gamma(-\imath \xi / a) d \xi(27)
$$

In the second equality on the right hand side in (27) we have substituted for $e^{\zeta}$ and used the integral representation $\rho(\xi)=1 /(2 \pi) \int_{-\infty}^{\infty} \exp \left(-\imath \xi-|\xi|^{\mu}\right) d \xi$ of the Lévy distribution. We then have changed integration limits in one of the integrals by making use of the Cauchy

theorem from complex analysis and replaced one of the integrals by the Gamma function making use of its integral representation. The minus sign in the last expression in (27) stems from changing the integration limits after applying the Cauchy theorem. In further calculations we exploit the following results:

\section{Laplace Transforms:}

$$
\begin{aligned}
& \mathcal{L}_{t}\left[t^{\alpha}\right](s)=\Gamma(\alpha+1) / s^{\alpha+1} \\
& \mathcal{L}_{t}\left[e^{\imath k t}\right](s)=1 /(s-\imath k) \\
& \mathcal{L}_{t}[f(k t)](s)=1 / k \mathcal{L}_{t}[f(t)](s / k) \\
& \mathcal{L}_{t}\left[\frac{1}{(\ln (t))^{n}}\right](s)=\frac{(-1)^{n}}{s(\ln (s))^{n}} \sum_{p=0}^{\infty}(-1)^{p} \frac{\mathfrak{k}_{p}}{(\ln (s))^{p}} \\
& \mathcal{L}_{t}\left[\frac{e^{-\alpha t}}{(\ln (k t))^{n}}\right](s)=\frac{1}{(\alpha+s)} \frac{1}{\left(\ln \left(\frac{k}{s}\right)\right)^{n}} \sum_{p=0}^{\infty} \frac{\mathcal{C}_{p}}{\left(\ln \left(\frac{k}{s}\right)\right)^{p}}
\end{aligned}
$$

\section{The Gamma Function}

$$
\Gamma(x) \Gamma(1-x)=\pi / \sin (\pi x) \quad \text { for } \quad x \in \mathbb{C}
$$

\section{Convolutions of Laplace transforms}




$$
\begin{aligned}
& \left(\frac{1}{s} \otimes \frac{1}{s}\right)=\frac{1}{s} \\
& \left(s^{n} \otimes \frac{1}{s(\ln (s))^{m}}\right)=\frac{s^{n}}{(\ln (s))^{m}} \sum_{p=0}^{\infty} \mathfrak{O}_{m, p} \frac{\mathfrak{m}_{p}}{(\ln (s))^{p}} \\
& \left(\frac{1}{s(\ln (s))^{n}} \otimes \frac{1}{s(\ln (s))^{m}}\right)=\frac{1}{s(\ln (s))^{n+m}} \sum_{p=0}^{\infty} \mathfrak{O}_{m, p} \frac{\mathfrak{n}_{p}}{(\ln (s))^{p}}
\end{aligned}
$$

where $\mathfrak{m}_{p}:=\sum_{q=1}^{n}(-1)^{q} / q^{p+1}, \mathfrak{n}_{p}:=\sum_{q=1}^{\infty} \mathfrak{l}_{q}(n) / q^{p+1}$ and $\mathfrak{O}_{m, p}:=(m+p-1) ! /(m-1) !$. Here $\otimes$ denotes a convolution $(f(s) \otimes g(s))(s):=\int_{0}^{s} f(\xi) g(s-\xi) d \xi$ and the remaining parameters are defined and the results are derived in Appendix.

Now we work out the Laplace transform of the function $\mathfrak{f}(t)$. We get:

$$
\tilde{\mathfrak{f}}(s)=\mathcal{L}_{t}[\mathfrak{f}(t)](s)=\frac{1}{2}(\tilde{\mathfrak{F}}(1, s)+\tilde{\mathfrak{F}}(-\Sigma, s))
$$

where

$$
\begin{aligned}
& \tilde{\mathfrak{F}}(a, s)=\frac{-1}{2 \pi a} \int_{-\infty}^{\infty} \exp \left(-|\xi|^{\mu}+\pi /(2 a) \xi\right) \frac{1}{s^{1+\imath \xi / a}} \frac{\imath \pi}{\sinh (\pi \xi / a)} d \xi \\
& =\frac{1}{2 a s} \int_{-\infty}^{\infty} \exp \left(-|\xi|^{\mu}+\pi /(2 a) \xi\right) \frac{\sin (\xi / a \ln (s))}{\sinh (\pi \xi / a)} d \xi \\
& =\frac{1}{2 a s} \int_{-\infty}^{\infty} \exp \left(-|\xi|^{\mu}+\pi /(2 a) \xi\right) \cdot \frac{\xi}{\sinh (\pi \xi / a)} \cdot \frac{\sin (\xi(\ln (s) / a))}{\xi} d \xi
\end{aligned}
$$

In the first equality (38) we make use of (28) and of the result (33) concerning a product of Gamma functions. In (39) we write the term $s^{\imath \xi / a}$ as $\exp (\imath \xi / a \ln (s))$ and we disregard the imaginary part owing to the fact that the result is real. In (40) we separate the integrand into a product of terms that will be handled later on.

Now we find a small-s approximation to the function $\tilde{\mathfrak{F}}(a, s)$. Since for small positive $s$ the last part of the integrand in (40) is a very "high frequency" periodic function of $\xi$ with a maximum $(\ln (s) / a)$ at $\xi=0$ and a period of length $2 \pi a /|\ln (s)|$ we apply the following approximations (to be termed steepest descent approximations) for our calculations.

$\mathfrak{b}(1)$ Expand the first term $\mathfrak{I}(\xi):=\exp \left(-|\xi|^{\mu}+\pi /(2 a) \xi\right)$ in the integrand (40) in a Taylor series in $\xi$ to the second order around zero. Note that the maximum of the whole integrand (the product of all three terms in (40)) is bigger than zero but, since the expression $\sin (\xi(\ln (s) / a)) / \xi$ behaves for small positive $s$ 
as $\delta(\xi / a)$, the maximum of the whole integrand will be arbitrarily close to zero for sufficiently small $s$.

$\mathfrak{b}(2)$ Confine the integration range to the first period $[-\pi a /|\ln (s)|, \pi a /|\ln (s)|]$ of the "high-frequency" periodic function in the last term of the integrand.

$\mathfrak{b}(3)$ Replace the middle and the last terms in the integrand by a small $\xi$ expansion around $\xi=0$.

This yields a following result:

$$
\begin{aligned}
\tilde{\mathfrak{F}}(a, s) & \simeq \frac{1}{2 a s} \int_{-\pi a /|\ln (s)|}^{\pi a /|\ln (s)|}\left(\mathfrak{I}(0)+\frac{1}{2} \mathfrak{I}^{\prime \prime}\left(0_{+}\right) \xi^{2}\right) \cdot \frac{a}{\pi} \cdot \frac{\ln (s)}{a} d \xi \\
& =\frac{1}{2 a s} \cdot \frac{\ln (s)}{\pi} \cdot\left[\mathfrak{I}(0) \frac{2 \pi a}{|\ln (s)|}+\frac{1}{3} \mathfrak{I}^{\prime \prime}\left(0_{+}\right)\left(\frac{\pi a}{|\ln (s)|}\right)^{3}\right] \\
& =\frac{1}{s}-\frac{\pi^{2}}{6}\left|\mathfrak{I}^{\prime \prime}\left(0_{+}\right)\right| a^{2} \frac{1}{s \ln (s)^{2}}+\frac{1}{s} O\left(\frac{1}{\ln (s)^{4}}\right)
\end{aligned}
$$

This calculation is incomplete in that we did not explain sufficiently the nature of approximation used by computation of the integral. In fact we should have expanded the integrand around its maximum $\xi_{0}=\xi_{0}(s)$, calculated the integral and only then considered the result in the limit of small positive $s$. This would only change the value of the parameter $\mathfrak{I}^{\prime \prime}\left(0_{+}\right)$in the result. In our calculations this parameter is left unknown.

Now we invert the Laplace transform $\tilde{\mathfrak{f}}(s)$ and, on the grounds of (31) and of (37), we get:

$$
\tilde{\mathfrak{f}}(t)=\mathcal{L}_{s}^{-1}[\tilde{\mathfrak{f}}(s)](t)=1-\frac{\mathfrak{K}}{(\ln (t))^{2}}+O\left(\frac{1}{(\ln (t))^{4}}\right)
$$

where $\mathfrak{K}$ is some parameter that is unknown. Inserting (42) into (24) we obtain the jump probability in the limit of large times:

$$
\begin{aligned}
\tilde{\omega}_{t}(k, t) & =\frac{1}{2 \pi} e^{-\imath k \int_{0}^{t} \mathcal{H}_{d}(\xi) d \xi} \cdot \lim _{N \rightarrow \infty}\left[1-\frac{\mathfrak{K}}{\left(\ln \left(\frac{k t}{\varpi(N)}\right)\right)^{2}}\right]^{N} \\
& =\frac{1}{2 \pi} e^{-\imath k \int_{0}^{t} \mathcal{H}_{d}(\xi) d \xi} \cdot \lim _{N \rightarrow \infty} \exp \left\{-\frac{N \mathfrak{K}}{\left(\ln \left(\frac{k t}{\varpi(N)}\right)\right)^{2}}\right\} \\
& =\frac{1}{2 \pi} e^{-\imath k \int_{0}^{t}(\Delta(\xi)+2) d \xi} \cdot \exp \left\{-\frac{\mathfrak{K}}{(\ln (k t))^{2}}\right\}
\end{aligned}
$$


where in (45) we have chosen the number of subintervals $\varpi(N)$ as $\varpi(N)=(k t)^{\sqrt{N}+1}$ to ensure that the limit is finite. Now we use result (32) to calculate the Fourier-Laplace transform $\tilde{\omega}_{t}(k, s)$ of the jump pdf and to obtain the FourierLaplace transform of the occupational probability $\tilde{P}(k, s)$ of the discounted bond price from (16). We assume for simplicity that the function $\Delta(\xi)$ does not depend on time.

$$
\begin{aligned}
& \tilde{\omega}_{t}(k, s)=\mathcal{L}_{t}\left[e^{-\imath k \mathcal{D} t} \cdot \exp \left\{-\frac{\mathfrak{K}}{(\ln (k t))^{2}}\right\}\right](s) \\
& =\sum_{n=0}^{\infty} \frac{(-\mathfrak{K})^{n}}{n !} \mathcal{L}_{t}\left[\frac{e^{-\imath k \mathcal{D} t}}{(\ln (k t))^{2 n}}\right](s) \\
& =\frac{1}{(\imath k \mathcal{D}+s)}\left(1-\mathfrak{K} \frac{1}{\left(\ln \left(\frac{k}{s}\right)\right)^{2}}+O\left(\frac{1}{\left(\ln \left(\frac{k}{s}\right)\right)^{3}}\right)\right)
\end{aligned}
$$

where $\mathcal{D}:=(\Delta+2)$. Inserting $k=0$ in $(48)$ we get $\tilde{\phi}(s)=\tilde{\omega}_{t}(0, s)=1 / s$ what implies that the waiting time pdf $\phi(t)$ is constant $\phi(t)=1$ as a function of time and is not normalisable $\int_{0}^{\infty} \phi(t) d t=\infty$ ! Because of this constructing the occupational probability $\mathcal{P}(x, t)$ from $(16)$ in a process where the transition of the walker to $x$ in time $t$ consists of performing a certain number of jumps and of being immobile for some time may be impossible. This is because the Fourier Laplace transform $\tilde{\omega}_{t}(k, s)$ may be bigger than one and thus the formula (16) cannot be applied.

Note that if the drift $\Delta(\xi)$ satisfies a condition: $\Delta+2=0$ then the Fourier transform of the jump pdf decays as $\tilde{\omega}_{t}(k, t) \sim \exp \left\{-\mathfrak{K} /(\ln (k t))^{2}\right\}$ This decay is much slower than the inverse of any positive power of $t$. The interpretation of this result and fitting the model to high-frequency financial data is left for future work.

\section{Master equation for the discounted bond price}

In this section we derive a partial differential equation that is satisfied by the jump pdf $\omega_{t}(x, t)$. The derivation bases on the representation (21) of the jump pdf as a path integral. Note that equation (24) can be written as follows:

$$
\tilde{\omega}_{t}\left(k, t-t^{\prime}\right)=\frac{1}{2 \pi} \exp \left[-\imath k \int_{t^{\prime}}^{t}(\Delta(\xi)+2) d \xi\right] \cdot \exp \left[-\int_{t^{\prime}}^{t} \tilde{\mathfrak{H}}^{(\Sigma(\xi))}(k) d \xi\right]
$$


where

$$
\tilde{\mathfrak{H}}^{(\Sigma)}(k):=-\ln (\mathfrak{f}(k))=\ln \left[\int_{-\infty}^{\infty} \exp \left\{\imath k\left(e^{\zeta}+e^{-\Sigma \zeta}\right)\right\} \rho(\zeta) d \zeta\right]
$$

The function $\tilde{\mathfrak{H}}^{(\Sigma)}(k)$ can be viewed as a "log-characteristic function" related to a certain integral transformation of the jump pdf $\rho(\zeta)$, a transformation defined by equation (25). If we now assume $\Delta(\xi)$ and $\Sigma(\xi)$ to be constant as functions of $\xi$ then from (49) we obtain an equation for the Fourier transform of the jump pdf

$$
\partial_{t} \tilde{\omega}_{t}(k, t)=(\Delta+2)(-\imath k) \tilde{\omega}_{t}(k, t)-\tilde{\mathfrak{H}}^{(\Sigma)}(k) \tilde{\omega}_{t}(k, t)
$$

and, after taking the inverse Fourier transform, an equation for the jump pdf itself:

$$
\partial_{t} \omega_{t}(x, t)=-(\Delta+2) \partial_{x} \omega_{t}(x, t)-\tilde{\mathfrak{H}}^{(\Sigma)}\left(-\imath \partial_{x}\right) \omega_{t}(x, t)
$$

where the operator $\tilde{\mathfrak{H}}^{(\Sigma)}\left(-\imath \partial_{x}\right)$ is defined by expanding the function $\tilde{\mathfrak{H}}^{(\Sigma)}(k)$ in a Taylor series around $k=0$ and by replacing $k$ by $-\imath \partial_{x}$. The master equation (52) corresponds to formula (112) on page 17 in Kleinert (2002).

In future work we will check if the master equation (52) includes fractional derivatives. In particular we will investigate if it can be related to distributed order fractional diffusion equations Chechkin (2002); Gorenflo (2003); Sokolov (2004); Meerschaert (2004); Scheffler (2004) that are widely studied due to their connection to ultraslow diffusion.

\section{Conclusions}

We derived the large time limit of a Continuous Time Random Walk where the motion of the walker is driven by a stochastic process with Paretian fluctuations. We applied the CTRW model to the Heath-Jarrow-Morton model for the term structure of interest rates and showed that if the forward rate of interest is driven by Paretian fluctuations then the bond price follows a stochastic process that behaves like a biased random walk. This means that in our model arbitrage opportunities cannot be canceled out by an appropriate choice of the drift and the volatility parameters of the HJM model. Further work will be devoted to deriving asymptotics of distributions of first hitting times in the CTRW model and to developing a theory of pricing options in the Black and Scholes framework. 
At the end of writing the manuscript we became aware of a different work [ Eberlein Raible (1999) ] devoted to the subject matter. We will refer to that paper in our future work.

\section{Appendix}

In this section we derive mathematical results regarding the small-s expansion (36) for a convolution of Laplace transforms, and Laplace transforms (31), (32) of a power of an inverse logarithm and of a function that involves exponentials and powers of inverse logarithms.

At first we compute the convolution that is used for computing the Laplace transform of the path integral (21). Here:

$$
\begin{aligned}
\mathfrak{s} & :=\left(\frac{1}{s(\ln (s))^{n}} \otimes \frac{1}{s(\ln (s))^{m}}\right)=\int_{0}^{s} \frac{d \xi}{\xi(\ln (\xi))^{m}} \frac{1}{(s-\xi)(\ln (s-\xi))^{n}} \\
& =\int_{-\infty}^{\ln (s)} \frac{d z}{z^{m}} \frac{1}{\left(s-e^{z}\right)\left(\ln \left(s-e^{z}\right)\right)^{n}}=\frac{1}{s(\ln (s))^{n}} \int_{-\infty}^{\ln (s)} \frac{d z}{z^{m}} \mathfrak{L}^{(n)}\left(e^{z-\ln (s)}\right) \\
& =\frac{(-1)^{m}}{s(\ln (s))^{n}} \int_{-\ln (s)}^{\infty} \frac{d w}{w^{m}} \mathfrak{L}^{(n)}\left(\frac{e^{-w}}{s}\right)
\end{aligned}
$$

where in $(53)=(54)$ we substituted for $\ln (\xi)$, then we introduced an auxiliary function $\mathfrak{L}^{(n)}(x):=(1-x)^{-1}(1+\ln (1-x) / \ln (s))^{-n}$ and finaly in $(54)=(55)$ we substited for $-z$. Now we expand the function $\mathfrak{L}^{(n)}$ in a Taylor series $\mathfrak{L}^{(n)}(x)=\sum_{p=0}^{\infty} \mathfrak{l}_{p}(n) x^{p}$, insert the expansion into (55) and we get:

$$
\mathfrak{s}=\frac{(-1)^{m}}{s(\ln (s))^{n}} \sum_{p=1}^{\infty} \frac{p^{m-1} \mathfrak{l}_{p}(n)}{s^{p}} \int_{-p \ln (s)}^{\infty} w^{-m} \exp (-w) d w
$$

We recall that the integrals (56) are integral representations of the truncated Gamma function

$$
\Gamma(x, m):=\int_{x}^{\infty} w^{-m} \exp (-w) d w
$$

which has a following asymptotic expansion:

$$
\Gamma(x, m)=\exp (-x) \sum_{q=0}^{\infty}(-1)^{q} \frac{(m+q-1) !}{(m-1) !} \frac{1}{x^{m+q}}
$$


Inserting the expansion (58) into (56) we obtain the result (36) q.e.d. Note that the coefficients $\mathfrak{l}_{p}(n)$ in the expansion of the function $\mathfrak{L}^{(n)}$ also depend on $s$. They have a form: $\mathfrak{l}_{p}(n)=\mathfrak{P}_{p}^{(n)}(1 / \log (s))$ where $\mathfrak{P}_{p}^{(n)}$ are polynomials of order $p$ and $\mathfrak{P}_{p}^{(n)}=1$.

Now we derive the Laplace transform (32) of a function that involves an exponential and powers of the inverse natural logarithm. We calculate:

$$
\mathcal{L}_{t}\left[\frac{e^{-\alpha t}}{(\ln (k t))^{n}}\right](s)=\frac{1}{k}\left(\mathcal{I}_{n}^{-}\left(\frac{\alpha+s}{k}, a\right)+\mathcal{I}_{n}^{+}\left(\frac{\alpha+s}{k}, a\right)\right)
$$

where

$$
\mathcal{I}_{n}^{-}(\alpha, a):=\int_{0}^{a} \frac{\exp (-\alpha t)}{(\ln (t))^{n}} d t \quad \text { and } \quad \mathcal{I}_{n}^{+}(\alpha, a):=\int_{a}^{\infty} \frac{\exp (-\alpha t)}{(\ln (t))^{n}} d t
$$

In the following we compute the integrals $\mathcal{I}_{+}^{(n)}(\alpha, a)$ and $\mathcal{I}_{-}^{(n)}(\alpha, a)$. For computing the integral $\mathcal{I}_{+}^{(n)}(\alpha, a)$ we consider a following auxiliary integral:

$$
\mathcal{J}_{n, p}(\alpha, a):=\int_{a}^{\infty} \frac{\exp (-\alpha t)}{(\ln (t))^{n} t^{p}} d t \quad \text { such that } \quad \mathcal{I}_{n}^{+}(\alpha, a)=\mathcal{J}_{n, 0}(\alpha, a)
$$

The integral $\mathcal{J}_{n, p}(\alpha, a)$ differentiated with respect to the parameter $p$ yields the truncated Gamma function (57). We have

$$
\begin{aligned}
& \frac{d^{n} \mathcal{J}_{n, p}(\alpha, a)}{d p^{n}}=\int_{a}^{\infty} e^{-\alpha t} \frac{(-\ln (t))^{n}}{(\ln (t))^{n}} \frac{d t}{t^{p}} \\
& =(-1)^{n} \alpha^{p-1} \Gamma(\alpha a, p)=(-1)^{n} \frac{e^{-\alpha a}}{\alpha} \sum_{q=0}^{\infty} \frac{(-1)^{q}}{(\alpha a)^{q}} \frac{(p+q-1) !}{(p-1) !} e^{-p \ln (a)}
\end{aligned}
$$

where on the right hand side in (62) we used the identity $1 / t^{p}=\exp (-p \ln (t))$ and in (63) we used the asymptotic expansion (58) of the truncated Gamma function. Integrating (63) $n$ times with respect to $p$ from $p$ to infinity and using the fact $(61)$ that $d^{m} \mathcal{J}_{n, p}(\alpha, a) /\left.d p^{m}\right|_{p=\infty}=0$ for $m=0, \ldots, n$ we get:

$$
\mathcal{J}_{n, p}(\alpha, a)=(-1)^{n} \frac{e^{-\alpha a}}{\alpha} \sum_{q=0}^{\infty} \frac{(-1)^{q}}{(\alpha a)^{q}} F_{p}^{(n)}\left[e^{-\xi \ln (a)} \prod_{s=0}^{q-1}(\xi+s), \xi\right]
$$


where

$$
F_{p}^{(n)}[f(\xi), \xi]:=\int_{p}^{\infty} \int_{\xi_{1}}^{\infty} \ldots \int_{\xi_{n-1}}^{\infty} f\left(\xi_{n}\right) d \xi_{n} \cdot \ldots \cdot d \xi_{2} d \xi_{1}=\frac{1}{(n-1) !} \int_{p}^{\infty} f(\xi) \xi^{n-1} d \xi(65)
$$

We insert the last expression on the right hand side in (65) into (64), we evaluate the integral over $\xi$ by successive integration by parts and we obtain:

$$
\mathcal{J}_{n, p}(\alpha, a)=\frac{(-1)^{n}}{(n-1) !} \frac{e^{-\alpha a}}{a^{p} \alpha} \sum_{q=0}^{\infty} \frac{(-1)^{q}}{(\alpha a)^{q}} \sum_{l=0}^{n+q-1} \frac{W_{n-1, q}^{(l)}(p)}{(\ln (a))^{l+1}}
$$

where

$$
W_{n-1, q}(\xi):=\xi^{n-1} \prod_{s=0}^{q-1}(\xi+s)
$$

and $W_{n-1, q}^{(l)}(p)$ denotes the $l$ th derivative evaluted at $\xi=p$.

Now we set $p=0$. Since the polynomial $W_{n-1, q}(\xi)$ has $(67)$ a leading power $\xi^{n-1}$ then the derivatives $W_{n-1, q}^{(l)}(0)$ for $l=0, \ldots, n-2$ at zero are equal to zero. This allows to transform the expression (66) for $p=0$ to the following form:

$$
\begin{aligned}
& \mathcal{J}_{n, 0}(\alpha, a)=\mathcal{I}_{n}^{+}(\alpha, a)= \\
& \frac{(-1)^{n}}{(n-1) !} \frac{e^{-\alpha a}}{\alpha} \frac{1}{(\ln (a))^{n}} \sum_{l=0}^{\infty} \frac{1}{(\ln (a))^{l}} \sum_{q=l}^{\infty} \frac{W_{n+q-1}^{(n+l-1)}(0)}{(\alpha a)^{q}}(-1)^{q}
\end{aligned}
$$

It is easy to work out the form of the coefficients $W_{n+q-1}^{(n+l-1)}(0)$. They take the form

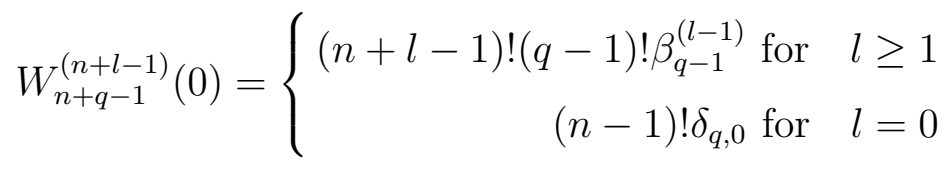

where

$$
\beta_{r}^{(l)}:=\sum_{1 \leq \xi_{1}<\ldots<\xi_{l} \leq r}\left(\xi_{1} \cdot \ldots \cdot \xi_{l}\right)^{-1} \quad \text { for } l \geq 1 \text { and } \quad \beta_{r}^{(0)}=1
$$

Inserting (69) into (68) and changing the summation index $q=l+r$ to $r$ we 
get:

$$
\mathcal{I}_{n}^{+}(\alpha, a)=(-1)^{n} \frac{e^{-\alpha a}}{\alpha} \frac{1}{(\ln (a))^{n}}\left[\sum_{l=0}^{\infty}\left(\begin{array}{c}
n+l-1 \\
l
\end{array}\right) \frac{\mathcal{A}_{l}(\alpha a)}{(\ln (a))^{l}}\right]
$$

where

$$
\mathcal{A}_{l}(a):=\sum_{r=0}^{\infty} \frac{l !(l+r-1) !}{(a)^{l+r}} \beta_{l+r-1}^{(l-1)}(-1)^{l+r} \quad \text { and } \quad \mathcal{A}_{0}(a)=1
$$

Now we calculate the integral $\mathcal{I}_{n}^{-}(\alpha, a)$. The procedure is similar to that used in the first part of the appendix for deriving result (36). The integral reads:

$$
\begin{aligned}
& \mathcal{I}_{n}^{-}(\alpha, a)=(-1)^{n} \int_{-\ln (a)}^{\infty} \exp \left(-\alpha e^{-z}\right) \frac{e^{-z}}{z^{n}} d z= \\
& (-1)^{n} \sum_{l=0}^{\infty} \frac{(-\alpha)^{l}}{l !}(l+1)^{n-1} \Gamma(-(l+1) \ln (a), n)= \\
& (-1)^{n} \sum_{l=0}^{\infty} \frac{(-\alpha)^{l}}{l !}(l+1)^{n-1} a^{l+1} \sum_{q=0}^{\infty} \frac{(n+q-1) !}{(n-1) !} \frac{(-1)^{q}}{(-(l+1) \ln (a))^{n+q}}= \\
& \frac{1}{(\ln (a))^{n}} \sum_{l=0}^{\infty} \frac{(-\alpha)^{l}}{(l+1) !} a^{l+1} \sum_{q=0}^{\infty} \frac{(n+q-1) !}{(n-1) !} \frac{1}{((l+1) \ln (a))^{q}}= \\
& \frac{1}{(\ln (a))^{n}} \sum_{l=0}^{\infty} \frac{(n+l-1) !}{(n-1) !} \frac{1}{(\ln (a))^{l}} \sum_{q=0}^{\infty} \frac{(-\alpha)^{q}}{q !} \frac{a^{q+1}}{(q+1)^{l+1}}
\end{aligned}
$$

where in (73) we substituted for $-\ln (t)$, in (74) we expanded the exponential $\exp \left(-\alpha e^{-z}\right)$ in a series of $e^{-z}$ and transformed the resulting integrals to the truncated Gamma function, in (75) we made use of the asymptotic expansion (58) of the truncated Gamma function and finaly in (76) and in (77) we collected together terms with the same power of the inverse logarithm.

$$
\mathcal{I}_{n}^{-}(\alpha, a)=\frac{1}{\alpha(\ln (a))^{n}}\left[\sum_{l=0}^{\infty}\left(\begin{array}{c}
n+l-1 \\
l
\end{array}\right) \frac{\mathcal{B}_{l}(\alpha a)}{(\ln (a))^{l}}\right]
$$

where

$$
\mathcal{B}_{l}(a)=l ! \sum_{q=0}^{\infty} \frac{(-1)^{q}}{q !} \frac{(a)^{q+1}}{(q+1)^{l+1}}=\int_{0}^{a}\left(\ln \left(\frac{a}{z}\right)\right)^{l} e^{-z} d z
$$


The proof of the last equality in (79) is left to the reader. Having derived the results (71) and (78) we complete the computation of the Laplace transform (59). The result obviously depends on the parameter $a$ that divides the integration domain into two parts. Since we are interested in the small $s$ limit of the Laplace transform we choose $a$ to be a decreasing function of $s$, e.g. $a=k / s$. This conforms to the intuition since $a$ has to be large enough for the asymptotic series in (72) to converge. We obtain the following result:

$$
\mathcal{L}_{t}\left[\frac{e^{-\alpha t}}{(\ln (k t))^{n}}\right](s)=\frac{1}{(\alpha+s)} \frac{1}{\left(\ln \left(\frac{k}{s}\right)\right)^{n}} \sum_{l=0}^{\infty}\left(\begin{array}{c}
n+l-1 \\
l
\end{array}\right) \frac{\left(\mathcal{B}_{l}(\theta)+(-1)^{n} e^{-\theta} \mathcal{A}_{l}(\theta)\right)}{\left(\ln \left(\frac{k}{s}\right)\right)^{p}}(80)
$$

where $\theta=(\alpha+s) / s$. Therefore the proof of result (32) is finished. The Laplace transform (31) of a power of an inverse logarithm follows immediately by inserting $k=1$ and $\alpha=0$ into (80). The coefficients $\mathcal{C}_{p}$ and $\mathfrak{k}_{p}$ in (32) and (31) are expressed via coefficients $\mathcal{B}_{p}(\theta)$ and $\mathcal{A}_{p}(\theta)$ as follows:

$$
\begin{aligned}
\mathcal{C}_{p} & =(n+p-1) ! /(p !(n-1) !) \cdot\left(\mathcal{B}_{p}(\theta)+(-1)^{n} e^{-\theta} \mathcal{A}_{p}(\theta)\right) \\
\mathfrak{k}_{p} & =(n+p-1) ! /(p !(n-1) !) \cdot\left(\mathcal{B}_{p}(1)+(-1)^{n} e^{-1} \mathcal{A}_{p}(1)\right)
\end{aligned}
$$

\section{References}

Lucey B, Friday the 13th \& The Philosophical Basis of Financial Economics, Journal of Economics and Finance, Vol. 24, Fall 2000

Eberlein \& Raible, Term Structure Models Driven by General Lévy Processes, Mathematical Finance, 9, iss. 1, pp. 31-53 (1999)

Heath D et al, Bond pricing and the term structure of interest rates: a new methodology for contingent claims valuation, Econometrica, 60, No. 1, 77105 (1992)

Musiela M, Rutkowski M, Applications of Mathematics: Stochastic Modelling and applied probability, Springer-Verlag Berlin Heidelberg (1997)

Sornette D, "String" formulation of the dynamics of the forward interest rate curve European Physical Journal B, 3, n 1, p 125-37 (1998); preprints cond-mat/9802136 cond-mat/9801321

Karatsas I, Shreve S, Brownian Motion and Stochastic Calculus, 2nd ed. New York: Springer-Verlag, 1997; WWW: http://mathworld.wolfram.com/ItosLemma.html

Kleinert H, Option pricing for non-Gaussian price fluctuations, Physica A 338 n 1-2, 151-159 (2004)

Sornette D, $\phi^{q}$-field theory for portfolio optimization: "Fat Tails" and nonlinear correlations, Physics Reports 335, 19-92 (2000) 
Meerschaert M M, Scheffler H P, Limit Distributions for Sums of Independent Random Vectors: Heavy tails in Theory and Practice John Wiley \& Sons, Inc. 2001

Bielecki T, Rutkowski M, Modelling the Term Structure: Conditionally Markov Approach, IEE Transactions on Automatic Control, 49, No. 3 (2004)

Samko S.G., Kilbas A.A., Marichev O.I., Fractional Integrals and Derivatives, Theory and Applications, Gordon and Breach Publishers, London 1993

Redner S, Connection between First-Passage and Occupation Probabilities, in: A Guide To First-Passage Processes, Cambridge University Press (2001) pages $1-6$

Ernst M H, Nonlinear model-Boltzmann equations and exact solutions, Phys. Rep. (Review Section of Physics Letters) 78, No. 1, p 1-171 (1981)

Montroll E.W. et al., Random Walks on Lattices II, J. Math. Phys. 6 167-181 (1965)

Balescu R., Continuous Time Random Walks, in: Statistical Dynamics Matter out of Equilibrium Imperial College Press, World Scientific, London 1997

Chechkin A V, Gorenflo R, and Sokolov I M, Retarding subdiffusion and accelerating superdiffusion governed by distributed-order fractional diffusion equations. Phys Rev. E 66, 046129-046135 (2002)

Gorenflo R and Mainardi F, Fractional diffusion processes: Probability distribution and continuous time random walk. Preprint (2003)

Sokolov I M, Chechkin A V and Klafter J, Distributed-order fractional kinetics. Acta Physica Polonica B 35, 1323 (2004)

Meerschaert M M and Scheffler H P, Limit theorems for continuous time random walks with slowly varying waiting times. http://unr.edu/homepage/mcubed//ultraslow.pdf (2004)

Meerschaert M M and Scheffler H P, Stochastic model for ultraslow diffusion http://unr.edu/homepage/mcubed/ultraslow2.pdf (2004)

Kleinert H, Stochastic Calculus for Assets with Non-Gaussian Price Fluctuations, preprint cond-mat/0203157 